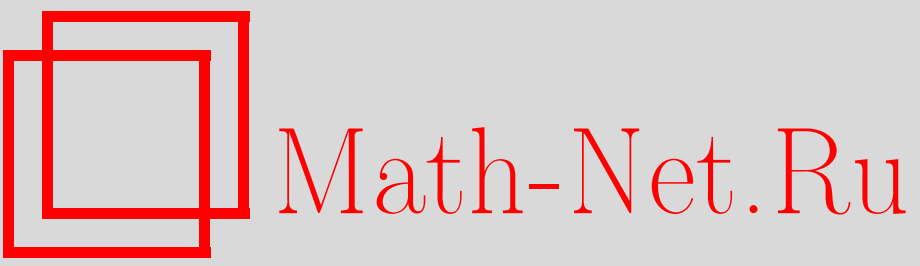

Н. И. Гусева, Е. В. Лукьянова, Композиционные алгебры произвольных степеней, Итоги науки и техн. Сер. Соврем. мат. и ее прил. Темат. обз., 2020, том 181, 16-21

DOI: https://doi.org/10.36535/0233-6723-2020-181-16-21

Использование Общероссийского математического портала Math-Net.Ru подразумевает, что вы прочитали и согласны с пользовательским соглашением

http://www.mathnet.ru/rus/agreement

Параметры загрузки :

IP: 54.89 .56 .158

26 апреля 2023 г., 15:50:32 


\title{
КОМПОЗИЦИОННЫЕ АЛГЕБРЫ ПРОИЗВОЛЬНЫХ СТЕПЕНЕЙ
}

\author{
(c) 2020 г. $\quad$ Н. И. ГУСЕВА, Е. В. ЛУКЬЯНОВА
}

\begin{abstract}
АннотАция. В статье дан обобщение композиционных алгебр, в котором квадратичная форма заменяется формой произвольной степени. Найдены две серии обобщенных композиционных алгебр: циклические алгебры произвольного порядка и алгебры, которые получаются обобщенной процедурой удвоения, примененной к циклическим алгебрам.
\end{abstract}

Ключевые слова: композиционная алгебра, проблема Гурвица, циклическая алгебра, процедура удвоения.

\section{COMPOSITION ALGEBRAS OF ARBITRARY DEGREES}

\author{
(C) 2020 N. I. GUSEVA, E. V. LUKYANOVA
}

\begin{abstract}
In this paper, a generalization of composition algebras is given, in which the quadratic form is replaced by a form of arbitrary degree. Two series of generalized composition algebras are found: cyclic algebras of arbitrary order and algebras that are obtained by the generalized doubling procedure applied to cyclic algebras.
\end{abstract}

Keywords and phrases: composition algebra, Hurwitz problem, cyclic algebra, doubling procedure.

AMS Subject Classification: 15A66, 15A69, 16S38

Хорошо известно, какую роль в математике и ее приложениях сыграло открытие У. Гамильтоном алгебры гиперкомплексных чисел, которые он назвал кватернионами. В алгебре это открытие привело к формированию понятия векторного пространства. Самым существенным приложением кватернионов в физике XIX в. стала формулировка Дж. Максвеллом уравнений электродинамики на основе кватернионного анализа (см. [1]). В XX в. алгебра комплексных кватернионов сыграла ключевую роль в развитии квантовой электродинамики (см. [6]).

Самым существенным положением теории кватернионов является наличие в этой алгебре невырожденной квадратичной формы, обладающей свойством мулътипликативности относительно произведения кватернионов. Такие алгебры называются композииионными; согласно теореме Гурвица, над полем вещественных чисел существуют (помимо самого поля) всего четыре ассоциативные композиционные алгебры (см. [4]): это алгебра комплексных чисел $\mathbb{C}$, алгебра двойных вещественных чисел $\mathbb{C}^{+}$, алгебра кватернионов $\mathbb{H}$ и алгебра антикватернионов $\mathbb{H}^{+}$. Над полем комплексных чисел (помимо самого поля) существует всего две композиционные алгебры: алгебра двойных комплексных чисел $\mathbb{C}_{2}$ и алгебра комплексных кватернионов $\mathbb{H}(\mathbb{C})$.

Алгебра кватернионов получается удвоением алгебры комплексных чисел: если взять две алгебры комплексных чисел $\mathbb{C}(i)$ и $\mathbb{C}(j)$ с мнимыми единицами $i$ и $j$, то кватернионами будут 
формальные линейные комбинации $z=z_{0}+z_{1} j$, где $z_{0}, z_{1} \in \mathbb{C}(i)$, умножение которых определяется следующим правилом:

$$
z a=\left(z_{0} a_{0}-z_{1} \bar{a}_{1}\right)+\left(z_{0} a_{1}+z_{1} \bar{a}_{0}\right) j .
$$

Такое умножение позволяет сконструировать квадратичную форму, мультипликативную относительно произведения кватернионов. Для этого заметим, что кватернионное линейное уравнение $z a=c$ эквивалентно системе вещественных линейных уравнений

$$
\left\{\begin{array}{l}
a_{0} z_{0}-\bar{a}_{1} z_{1}=c_{0} \\
a_{1} z_{0}+\bar{a}_{0} z_{1}=c_{1}
\end{array}\right.
$$

можно показать, что определитель этой системы

$$
\Delta_{2}(a)=\left|\begin{array}{rr}
a_{0} & -\bar{a}_{1} \\
a_{1} & \bar{a}_{0}
\end{array}\right|=a_{0} \bar{a}_{0}+a_{1} \bar{a}_{1}
$$

где $a_{0}=a_{00}+i a_{10}, a_{1}=a_{01}+i a_{11}$, является квадратичной формой

$$
a_{0} \bar{a}_{0}+a_{1} \bar{a}_{1}=a_{00}^{2}+a_{10}^{2}+a_{01}^{2}+a_{11}^{2},
$$

мультипликативной относительно умножения кватернионов. Это сразу следует из формулы (1) и правила умножения определителей:

$$
\Delta_{2}(z a)=\left|\begin{array}{rr}
z_{0} a_{0}-z_{1} \bar{a}_{1} & -\bar{z}_{0} \bar{a}_{1}-\bar{z}_{1} a_{0} \\
z_{0} a_{1}+z_{1} \bar{a}_{0} & \bar{z}_{0} \bar{a}_{0}-\bar{z}_{1} a_{1}
\end{array}\right|=\left|\begin{array}{rr}
a_{0} & -\bar{a}_{1} \\
a_{1} & \bar{a}_{0}
\end{array}\right|\left|\begin{array}{rr}
z_{0} & -\bar{z}_{1} \\
z_{1} & \bar{z}_{0}
\end{array}\right|=\Delta_{2}(a) \Delta_{2}(z) .
$$

Тождество (4), записанное в вещественных координатах

$$
\begin{gathered}
\left(a_{00} z_{00}-a_{10} z_{10}-a_{01} z_{01}-a_{11} z_{11}\right)^{2}+\left(a_{10} z_{00}+a_{00} z_{10}-a_{11} z_{01}+a_{01} z_{11}\right)^{2}+ \\
+\left(a_{01} z_{00}+a_{11} z_{10}+a_{00} z_{01}-a_{10} z_{11}\right)^{2}+\left(a_{11} z_{00}-a_{00} z_{10}+a_{10} z_{01}+a_{00} z_{11}\right)^{2}= \\
=\left(a_{00}^{2}+a_{10}^{2}+a_{01}^{2}+a_{11}^{2}\right)\left(z_{00}^{2}+z_{10}^{2}+z_{01}^{2}+z_{11}^{2}\right)
\end{gathered}
$$

называется тождеством четырех квадратов.

Аналогично, алгебра антикватернионов получается удвоением алгебры двойных вещественных чисел, а алгебра комплексных кватернионов - удвоением алгебры двойных комплексных чисел.

Вопрос о существовании композиционных алгебр называют проблемой Гурвица; теорема, носящая его имя, утверждает, что кроме перечисленных выше алгебр не существует других ассоциативных алгебр с невырожденной квадратичной формой, обладающей свойством мультипликативности относительно произведения элементов алгебры. Естественно возникает вопрос: существуют ли ассоциативные алгебры с невырожденной формой, не обязательно квадратичной, но обладающей свойством мультипликативности относительно произведения элементов данной алгебры? Такой вопрос можно назвать обобщенной проблемой Гурвица. Алгебры с невырожденной формой любой степени, обладающей свойством мультипликативности относительно произведения элементов данной алгебры, естественно называть обобщенно композиционными алгебрами.

Ответ на поставленный вопрос был дан М. П. Бурлаковым в конце 1970-х гг. (см. [2]). Он показал, что свойством мультипликативности обладают детерминанты элементов циклических и антициклических алгебр над полем вещественных чисел, а над полем комплексных чисел помимо комплексных циклических алгебр существуют обобщенно композиционные алгебры, которые при помощи обобщенной прочедуры удвоения получаются из циклических алгебр.

Комплексной циклической алгеброй порядка $m$ называется алгебра $\mathbb{C}_{m}(e)$, базисом которой будут элементы $e, e^{2}, \ldots, e^{m-1}$ и единица, а умножение определяется структурным тождеством $e^{m}=1$. Таким образом, произвольный элемент $x \in \mathbb{C}_{m}(e)$ имеет вид

$$
x=x_{0}+x_{1} e+x_{2} e^{2}+\ldots+x_{m-1} e^{m-1},
$$


где $x_{k} \in \mathbb{C}$, и для произведения элементов $x, a \in \mathbb{C}_{m}(e)$ получается тождество

$$
\begin{gathered}
x \cdot a=\left(x_{0}+x_{1} e+x_{2} e^{2}+\ldots+x_{m-1} e^{m-1}\right) \cdot\left(a_{0}+a_{1} e+a_{2} e^{2}+\ldots+a_{m-1} e^{m-1}\right)= \\
=\left(x_{0} a_{0}+x_{1} a_{m-1}+x_{2} a_{m-2}+\ldots+x_{m-1} a_{1}\right)+\left(x_{0} a_{1}+x_{1} a_{0}+x_{2} a_{m-1}+\ldots+x_{m-1} a_{2}\right) e+ \\
+\left(x_{0} a_{2}+x_{1} a_{1}+x_{2} a_{0}+\ldots+x_{m-1} a_{3}\right) e^{2}+\ldots+ \\
\quad+\left(x_{0} a_{m-1}+x_{1} a_{m-2}+x_{2} a_{m-3}+\ldots+x_{m-1} a_{0}\right) e^{m-1} .
\end{gathered}
$$

Из тождества (6) следует, что линейное алгебраическое уравнение $x \cdot a=c$ эквивалентно системе комплексных линейных уравнений с определителем следующего вида:

$$
\Delta_{m}(a)=\left|\begin{array}{ccccc}
a_{0} & a_{m-1} & a_{m-2} & \ldots & a_{1} \\
a_{1} & a_{0} & a_{m-1} & \ldots & a_{2} \\
a_{2} & a_{1} & a_{0} & \ldots & a_{3} \\
\vdots & \vdots & \vdots & \ddots & \vdots \\
a_{m-1} & a_{m-2} & a_{m-3} & \ldots & a_{0}
\end{array}\right| .
$$

Величина $\Delta_{m}(x)$ называется детерминантом элемента циклической алгебры $x \in \mathbb{C}_{m}(e)$ и представляет собой форму $m$-й степени, которая обладает мультипликативным свойством относительно произведения элементов этой алгебры, т.е.

$$
\Delta_{m}(x \cdot a)=\Delta_{m}(x) \Delta_{m}(a) .
$$

Доказательство тождества (8) получается прямым вычислением определителей входящих в это тождество. Например, для $m=2$

$$
\Delta_{2}(a \cdot x)=\left|\begin{array}{ll}
a_{0} x_{0}+a_{1} x_{1} & a_{1} x_{0}+a_{0} x_{1} \\
a_{1} x_{0}+a_{0} x_{1} & a_{0} x_{0}+a_{1} x_{1}
\end{array}\right|=\left|\begin{array}{cc}
a_{0} & a_{1} \\
a_{1} & a_{0}
\end{array}\right|\left|\begin{array}{cc}
x_{0} & x_{1} \\
x_{1} & x_{0}
\end{array}\right|=\Delta_{2}(a) \Delta_{2}(x)
$$

это тождество, записанное в комплексных координатах,

$$
\left(a_{0}^{2}-a_{1}^{2}\right)\left(x_{0}^{2}-x_{1}^{2}\right)=\left(a_{0} x_{0}+a_{1} x_{1}\right)^{2}-\left(a_{1} x_{0}+a_{0} x_{1}\right)^{2}
$$

называется тождеством двух квадратов. Впрочем, его чаще записывают, заменив $x_{1}$ на $i x_{1}$ и $a_{1}$ на $i a_{1}$ :

$$
\left(a_{0}^{2}+a_{1}^{2}\right)\left(x_{0}^{2}+x_{1}^{2}\right)=\left(a_{0} x_{0}-a_{1} x_{1}\right)^{2}+\left(a_{1} x_{0}+a_{0} x_{1}\right)^{2} .
$$

Выпишем еще композиционное тождество (8) для $m=3$ :

$$
\begin{aligned}
\Delta_{3}(x \cdot a)=\left|\begin{array}{lll}
x_{0} a_{0}+x_{1} a_{2}+x_{2} a_{1} & x_{0} a_{2}+x_{1} a_{1}+x_{2} a_{0} & x_{0} a_{1}+x_{1} a_{0}+x_{2} a_{2} \\
x_{0} a_{1}+x_{1} a_{0}+x_{2} a_{2} & x_{0} a_{0}+x_{1} a_{2}+x_{2} a_{1} & x_{0} a_{2}+x_{1} a_{1}+x_{2} a_{0} \\
x_{0} a_{2}+x_{1} a_{1}+x_{2} a_{0} & x_{0} a_{1}+x_{1} a_{0}+x_{2} a_{2} & x_{0} a_{0}+x_{1} a_{2}+x_{2} a_{1}
\end{array}\right|= \\
=\left|\begin{array}{lll}
a_{0} & a_{2} & a_{1} \\
a_{1} & a_{0} & a_{2} \\
a_{2} & a_{1} & a_{0}
\end{array}\right|\left|\begin{array}{ccc}
x_{0} & x_{2} & x_{1} \\
x_{1} & x_{0} & x_{2} \\
x_{2} & x_{1} & x_{0}
\end{array}\right|=\Delta_{3}(a) \Delta_{3}(x) .
\end{aligned}
$$

Это тождество, записанное в (комплексных) координатах, представляет собой кубический аналог тождества двух квадратов:

$$
\begin{aligned}
& \left(a_{0}^{3}+a_{1}^{3}+a_{2}^{3}-3 a_{0} a_{1} a_{2}\right)\left(x_{0}^{3}+x_{1}^{3}+x_{2}^{3}-3 x_{0} x_{1} x_{2}\right)= \\
& =\left(a_{0} x_{0}+a_{2} x_{1}+a_{1} x_{2}\right)^{3}+\left(a_{1} x_{0}+a_{0} x_{1}+a_{2} x_{2}\right)^{3}+\left(a_{2} x_{0}+a_{1} x_{1}+a_{0} x_{2}\right)^{3}- \\
& \quad-\left(a_{0} x_{0}+a_{2} x_{1}+a_{1} x_{2}\right)\left(a_{1} x_{0}+a_{0} x_{1}+a_{2} x_{2}\right)\left(a_{2} x_{0}+a_{1} x_{1}+a_{0} x_{2}\right)^{3} .
\end{aligned}
$$


Детерминанты вида (7) называются циркулянтами, и для их вычисления применяется следующая формула [5]:

$$
\Delta_{m}(x)=\prod_{k=0}^{m-1}\left(\sum_{h=0}^{m-1} \alpha_{m}^{k h} x_{h}\right),
$$

где $\alpha_{m}=\cos (2 \pi / m)+i \sin (2 \pi / m)$. Например, для $m=2$

$$
\Delta_{2}(x)=x_{0}^{2}-x_{1}^{2}=\left(x_{0}+x_{1}\right)\left(x_{0}-x_{1}\right)
$$

так как $\alpha_{2}=-1$, а для $m=3$ получается тождество

$$
\begin{aligned}
& \Delta_{3}(x)=x_{0}^{3}+x_{1}^{3}+x_{2}^{3}-3 x_{0} x_{1} x_{2}= \\
& =\left(x_{0}+x_{1}+x_{2}\right)\left(x_{0}^{2}+x_{1}^{2}+x_{2}^{2}-x_{0} x_{1}-x_{1} x_{2}-x_{2} x_{0}\right)= \\
& \quad=\left(x_{0}+x_{1}+x_{2}\right)\left(x_{0}+\alpha_{3} x_{1}+\alpha_{3}^{2} x_{2}\right)\left(x_{0}+\alpha_{3}^{2} x_{1}+\alpha_{3} x_{2}\right) .
\end{aligned}
$$

Формула (11) служит основанием для того, чтобы ввести в рассмотрение резолввенты для элементов $x \in \mathbb{C}_{m}(x)$, которые определяются следующим образом (см. [5]):

$$
x\left(\alpha_{m}^{k}\right)=x_{0}+x_{1} e^{k}+x_{2} e^{2 k}+\ldots+x_{m-1} e^{(m-1) k},
$$

и для единообразия считается, что $x(1)=x$. Прямым вычислением несложно показать, что $p e$ зольвентные операторы $\hat{\boldsymbol{\alpha}}_{m}^{k}: \mathbb{C}_{m}(e) \rightarrow \mathbb{C}_{m}(e)$, определенные формулой $\hat{\boldsymbol{\alpha}}_{m}^{k}(x)=x\left(\alpha_{m}^{k}\right)$, будут мультиинволютивными эндоморфизмами алгебры $\mathbb{C}_{m}(e)$, т.е. резольвентные операторы удовлетворяют следующим тождествам:

$$
\hat{\boldsymbol{\alpha}}_{m}^{k}(x+y)=\hat{\boldsymbol{\alpha}}_{m}^{k}(x)+\hat{\boldsymbol{\alpha}}_{m}^{k}(y), \quad \hat{\boldsymbol{\alpha}}_{m}^{k}(x \cdot y)=\hat{\boldsymbol{\alpha}}_{m}^{k}(x) \cdot \hat{\boldsymbol{\alpha}}_{m}^{k}(y), \quad \hat{\boldsymbol{\alpha}}_{m}^{m}(x)=x .
$$

При этом нетрудно видеть, что для $m=2$ резольвентный оператор совпадает с оператором сопряжения в алгебре двойных комплексных чисел $\mathbb{C}_{2}(e)$, т.е. резольвентные операторы представляют собой обобщение оператора сопряжения. Это обстоятельство приводит к конструкции обобщенной прочедуры удвоения циклических алгебр $\mathbb{C}_{m}(e)$ любого порядка.

По аналогии с классической процедурой удвоения, при помощи которой из алгебры комплексных чисел получалась алгебра кватернионов, а из алгебры двойных комплексных чисел - алгебра комплексных кватернионов, возьмем две циклические алгебры $\mathbb{C}_{m}\left(e_{1}\right)$ и $\mathbb{C}_{m}\left(e_{2}\right)$ и рассмотрим формальные линейные комбинации вида

$$
\boldsymbol{x}=x_{0}+x_{1} \cdot e_{2}+x_{2} \cdot e_{2}^{2}+\ldots+x_{m-1} \cdot e_{2}^{m-1}, \quad \text { где } \quad x_{k} \in \mathbb{C}_{m}\left(e_{1}\right) .
$$

Множество таких линейных комбинаций станет линейной алгеброй, если как-либо определить их умножение. В частности, умножение линейных комбинаций (13) можно определить коммутационными соотношениями вида

$$
e_{2} \cdot x=x\left(\alpha_{m}\right) \cdot e_{2}, \quad x \cdot e_{2}=e_{2} \cdot x\left(\alpha_{m}^{m-1}\right)
$$

для любых $x \in \mathbb{C}_{m}\left(e_{1}\right)$. Множество линейных комбинаций вида (13) с умножением, определенным коммутационными соотношениями $(14)$, образует линейную алгебру, которая обозначается $\mathbb{B}_{2}^{m}$ и называется алгеброй бионов $m$-го порядка, или двухмерной элементалъной алгеброй порядка $m$ (см. [2]).

Покажем, что алгебра $\mathbb{B}_{2}^{m}$ является обобщенно композиционной алгеброй $m$-го порядка, т.е. существуют форма $m$-й степени, мультипликативная относительно умножения элементов данной 
алгебры. Для этого вычислим произведение двух произвольных элементов $\boldsymbol{x}, \boldsymbol{y} \in \mathbb{B}_{2}^{m}$ :

$$
\begin{aligned}
\boldsymbol{x} \cdot \boldsymbol{y}=\left(x_{0}+\right. & \left.x_{1} \cdot e_{2}+x_{2} \cdot e_{2}^{2}+\ldots+x_{m-1} \cdot e_{2}^{m-1}\right) \times\left(y_{0}+y_{1} \cdot e_{2}+y_{2} \cdot e_{2}^{2}+\ldots+y_{m-1} \cdot e_{2}^{m-1}\right)= \\
& =\left(x_{0} \cdot y_{0}+x_{1} \cdot y_{m-1}\left(\alpha_{m}\right)+x_{2} \cdot y_{m-2}\left(\alpha_{m}^{2}\right)+\ldots+x_{m-1} \cdot y_{1}\left(\alpha_{m}^{m-1}\right)\right)+ \\
& +\left(x_{0} \cdot y_{1}+x_{1} \cdot y_{0}\left(\alpha_{m}\right)+x_{2} \cdot y_{m-1}\left(\alpha_{m}^{2}\right)+\ldots+x_{m-1} \cdot y_{2}\left(\alpha_{m}^{m-1}\right)\right) \cdot e_{2}+ \\
+ & \left(x_{0} \cdot y_{1}+x_{1} \cdot y_{1}\left(\alpha_{m}\right)+x_{2} \cdot y_{0}\left(\alpha_{m}^{2}\right)+\ldots+x_{m-1} \cdot y_{3}\left(\alpha_{m}^{m-1}\right)\right) \cdot e_{2}^{2}+\ldots \\
& \quad+\left(x_{0} \cdot y_{m-1}+x_{1} \cdot y_{m-2}\left(\alpha_{m}\right)+\ldots+x_{m-1} \cdot y_{0}\left(\alpha_{m}^{m-1}\right)\right) \cdot e_{2}^{m-1} \cdot(15)
\end{aligned}
$$

Это тождество подсказывает, что именно следует взять в качестве формы $m$-й степени, мультипликативной относительно умножения элементов алгебры $\mathbb{B}_{2}^{m}$. Обозначим

$$
\Delta_{m}(\boldsymbol{y})=\left|\begin{array}{ccccc}
y_{0} & y_{m-1}\left(\alpha_{m}\right) & y_{m-2}\left(\alpha_{m}^{2}\right) & \ldots & y_{1}\left(\alpha_{m}^{m-1}\right) \\
y_{1} & y_{0}\left(\alpha_{m}\right) & y_{m-1}\left(\alpha_{m}^{2}\right) & \ldots & y_{2}\left(\alpha_{m}^{m-1}\right) \\
y_{2} & y_{1}\left(\alpha_{m}\right) & y_{0}\left(\alpha_{m}^{2}\right) & \ldots & y_{3}\left(\alpha_{m}^{m-1}\right) \\
\vdots & \vdots & \vdots & \ddots & \vdots \\
y_{m-1} & y_{m-2}\left(\alpha_{m}\right) & y_{m-3}\left(\alpha_{m}^{2}\right) & \ldots & y_{0}\left(\alpha_{m}^{m-1}\right)
\end{array}\right|,
$$

и будем величину $\Delta_{m}(\boldsymbol{y})$ называть детерминантом элемента $\boldsymbol{y} \in \mathbb{B}_{2}^{m}$. Непосредственным вычислением можно показать, что

$$
\Delta_{m}(\boldsymbol{x} \cdot \boldsymbol{y})=\Delta_{m}(\boldsymbol{x}) \Delta_{m}(\boldsymbol{y}) .
$$

Например, для $m=3$ будем иметь

$$
\begin{gathered}
\Delta_{3}(\boldsymbol{x}) \Delta_{3}(\boldsymbol{y})=\left|\begin{array}{lll}
x_{0} & x_{2}\left(\alpha_{3}\right) & x_{1}\left(\alpha_{3}^{2}\right) \\
x_{1} & x_{0}\left(\alpha_{3}\right) & x_{2}\left(\alpha_{3}^{2}\right) \\
x_{2} & x_{1}\left(\alpha_{3}\right) & x_{0}\left(\alpha_{3}^{2}\right)
\end{array}\right| \cdot\left|\begin{array}{lll}
y_{0} & y_{2}\left(\alpha_{3}\right) & y_{1}\left(\alpha_{3}^{2}\right) \\
y_{1} & y_{0}\left(\alpha_{3}\right) & y_{2}\left(\alpha_{3}^{2}\right) \\
y_{2} & y_{1}\left(\alpha_{3}\right) & y_{0}\left(\alpha_{3}^{2}\right)
\end{array}\right| \\
=\left|\begin{array}{r}
x_{0} \cdot y_{0}+x_{2}\left(\alpha_{3}\right) \cdot y_{1}+x_{1}\left(\alpha_{3}^{2}\right) \cdot y_{2} \\
x_{1} \cdot y_{0}+x_{0}\left(\alpha_{3}\right) \cdot y_{1}+x_{2}\left(\alpha_{3}^{2}\right) \cdot y_{2} \\
x_{2} \cdot y_{0}+x_{1}\left(\alpha_{3}\right) \cdot y_{1}+x_{0}\left(\alpha_{3}^{2}\right) \cdot y_{2} \\
x_{0} \cdot y_{2}\left(\alpha_{3}\right)+x_{2}\left(\alpha_{3}\right) \cdot y_{0}\left(\alpha_{3}\right)+x_{1}\left(\alpha_{3}^{2}\right) \cdot y_{1}\left(\alpha_{3}\right) \\
x_{1} \cdot y_{2}\left(\alpha_{3}\right)+x_{0}\left(\alpha_{3}\right) \cdot y_{0}\left(\alpha_{3}\right)+x_{2}\left(\alpha_{3}^{2}\right) \cdot y_{1}\left(\alpha_{3}\right) \\
x_{2} \cdot y_{2}\left(\alpha_{3}\right)+x_{1}\left(\alpha_{3}\right) \cdot y_{0}\left(\alpha_{3}\right)+x_{0}\left(\alpha_{3}^{2}\right) \cdot y_{1}\left(\alpha_{3}\right) \\
x_{0} \cdot y_{1}\left(\alpha_{3}^{2}\right)+x_{2}\left(\alpha_{3}\right) \cdot y_{2}\left(\alpha_{3}^{2}\right)+x_{1}\left(\alpha_{3}^{2}\right) \cdot y_{0}\left(\alpha_{3}^{2}\right) \\
x_{1} \cdot y_{1}\left(\alpha_{3}^{2}\right)+x_{0}\left(\alpha_{3}\right) \cdot y_{2}\left(\alpha_{3}^{2}\right)+x_{2}\left(\alpha_{3}^{2}\right) \cdot y_{0}\left(\alpha_{3}^{2}\right) \\
x_{2} \cdot y_{1}\left(\alpha_{3}^{2}\right)+x_{1}\left(\alpha_{3}\right) \cdot y_{2}\left(\alpha_{3}^{2}\right)+x_{0}\left(\alpha_{3}^{2}\right) \cdot y_{0}\left(\alpha_{3}^{2}\right)
\end{array}\right|=\Delta_{3}(\boldsymbol{y} \cdot \boldsymbol{x}) .
\end{gathered}
$$

Остается показать, что хотя элементами определителя (16) являются элементы алгебры $\mathbb{C}_{2}\left(e_{1}\right)$, сам определитель будет комплексным числом. Доказательство этого факта следует из того, что элемент алгебры $\mathbb{C}_{m}\left(e_{1}\right)$ является комплексным числом тогда и только тогда, когда действие на него оператора резольвенты не меняет этот элемент. Несложно показать, что действие оператора резольвенты на $\Delta_{m}(\boldsymbol{y})$ не меняет этот определитель. Например,

$$
\begin{aligned}
\hat{\boldsymbol{\alpha}}_{3}\left(\Delta_{m}(\boldsymbol{y})\right)= & \hat{\boldsymbol{\alpha}}_{3}\left|\begin{array}{lll}
y_{0} & y_{2}\left(\alpha_{3}\right) & y_{1}\left(\alpha_{3}^{2}\right) \\
y_{1} & y_{0}\left(\alpha_{3}\right) & y_{2}\left(\alpha_{3}^{2}\right) \\
y_{2} & y_{1}\left(\alpha_{3}\right) & y_{0}\left(\alpha_{3}^{2}\right)
\end{array}\right|=\left|\begin{array}{lll}
y_{0}\left(\alpha_{3}\right) & y_{2}\left(\alpha_{3}^{2}\right) & y_{1} \\
y_{1}\left(\alpha_{3}\right) & y_{0}\left(\alpha_{3}^{2}\right) & y_{2} \\
y_{2}\left(\alpha_{3}\right) & y_{1}\left(\alpha_{3}^{2}\right) & y_{0}
\end{array}\right|=-\left|\begin{array}{lll}
y_{0}\left(\alpha_{3}\right) & y_{1} & y_{2}\left(\alpha_{3}^{2}\right) \\
y_{1}\left(\alpha_{3}\right) & y_{2} & y_{0}\left(\alpha_{3}^{2}\right) \\
y_{2}\left(\alpha_{3}\right) & y_{0} & y_{1}\left(\alpha_{3}^{2}\right)
\end{array}\right|= \\
& =\left|\begin{array}{lll}
y_{1} & y_{0}\left(\alpha_{3}\right) & y_{2}\left(\alpha_{3}^{2}\right) \\
y_{2} & y_{1}\left(\alpha_{3}\right) & y_{0}\left(\alpha_{3}^{2}\right) \\
y_{0} & y_{2}\left(\alpha_{3}\right) & y_{1}\left(\alpha_{3}^{2}\right)
\end{array}\right|=-\left|\begin{array}{lll}
y_{1} & y_{0}\left(\alpha_{3}\right) & y_{2}\left(\alpha_{3}^{2}\right) \\
y_{0} & y_{2}\left(\alpha_{3}\right) & y_{1}\left(\alpha_{3}^{2}\right) \\
y_{2} & y_{1}\left(\alpha_{3}\right) & y_{0}\left(\alpha_{3}^{2}\right)
\end{array}\right|=\left|\begin{array}{lll}
y_{0} & y_{2}\left(\alpha_{3}\right) & y_{1}\left(\alpha_{3}^{2}\right) \\
y_{1} & y_{0}\left(\alpha_{3}\right) & y_{2}\left(\alpha_{3}^{2}\right) \\
y_{2} & y_{1}\left(\alpha_{3}\right) & y_{0}\left(\alpha_{3}^{2}\right)
\end{array}\right|=\Delta_{m}(\boldsymbol{y}) .
\end{aligned}
$$

Таким образом, $\mathbb{B}_{2}^{m}$ дают серию обобщенно композиционных алгебр $m$-го порядка. Они получаются процедурой обобщенного удвоения циклических алгебр $m$-го порядка, подобно тому как алгебра комплексных кватернионов получается процедурой удвоения алгебры двойных комплексных 
чисел. Комплексная размерность алгебры $\mathbb{B}_{2}^{m}$ равна $m^{2}$, и любой ее элемент может быть записан в комплексных координатах следующей суммой:

$$
\boldsymbol{x}=\sum_{k=0}^{m-1} \sum_{h=0}^{m-1} x_{k h} e_{1}^{k} \cdot e_{2}^{h} .
$$

При этом очевидно, что $\mathbb{B}_{2}^{2}=\mathbb{H}(\mathbb{C})$, т.е. алгебры $\mathbb{B}_{2}^{m}$ обобщают алгебру комплексных кватернионов.

\section{СПИСОК ЛИТЕРАТУРЫ}

1. Александрова Н. В. Из истории векторного исчисления. - М.: МАИ, 1992.

2. Бурлаков М. П. Гамильтоновы алгебры. - М.: Граф-Пресс, 2006.

3. Гусева Н. И., Бурлаков И. М., Бурлаков М. П. Элементарная геометрия в пространствах над алгебрами. - М.: Интеллект-Центр, 2017.

4. Жевлаков К. А., Слинько А. М., Шестаков И. П., Ширшов А. И. Кольца, близкие к ассоциативным. - М.: Наука, 1978.

5. Проскурлков И. В. Сборник задач по линейной алгебре. - М.: Наука, 1967.

6. Хунд Ф. История квантовой теории. - Киев: Наукова думка, 1980.

Гусева Надежда Ивановна

Московский педагогический государственный университет;

Всероссийский институт научной и технической информации РАН, Москва

E-mail: ngus12@mail.ru

Лукьянова Елена Викторовна

Московский педагогический государственный университет

E-mail: lukyanovalv@list.ru 